\title{
Entre conflit et transaction sociale, une approche simmelienne aux défis des villes patrimonialisées
}

\author{
Les cas de Strasbourg et Valparaiso
}

\begin{abstract}
Maximiliano Soto ${ }^{1}$
[Résumé] En nous appuyant sur la sociologie de Georg Simmel, nous souhaitons éclairer les conflits du patrimoine bâti dans les villes patrimonialisées. Notre analyse porte sur deux villes dont les centres historiques sont inscrits au Patrimoine de l'Humanité par I'UNESCO, Strasbourg en France et Valparaiso au Chili. Ainsi nous appliquerons quelques éléments simmeliens pour développer une approche s'intéressant à la structure fonctionnelle de l'espace urbain patrimonial et aux éléments socio-affectifs dans les quartiers «historiques». Nous mettrons en avant la présence des conflits, d'échanges et des médiations des intérêts sociaux et économiques autour des biens patrimoniaux en suivant la notion simmelienne de conflit qui est abordée de manière positive en tant que productrice de liens sociaux. Dans les villes patrimonialisées, des intérêts pécuniaires publics et privés encouragent le développement des activités culturelles, tel que le tourisme culturel et l'amélioration des immeubles anciens. La notion de conflit chez Simmel permet donc de comprendre les effets des politiques d'aménagement et d'amélioration de l'espace urbain dit «patrimonial». En effet, les nouvelles formes de pouvoir et les processus transactionnels affectent les espaces territoriaux locaux par le biais de valorisations et de dévalorisations de l'espace urbain.
\end{abstract}

Mots-clés : conflit, transaction sociale, ville, patrimoine, formes patrimoniales.

[Abstract] Following the sociology of Georg Simmel, our aim is to clarify the conflicts of patrimony in heritage cities. We will focus on two cities whose historical centres are listed by UNESCO as a World Heritage, Strasbourg in France and Valparaiso in Chile. In that way we will apply some conceptions of Simmel's sociology in order to develop an approach which is interested in the functional structure of the human space of heritage and in the socio-emotional elements of "historical" neighbourhoods. We will point out the presence of conflicts, exchanges, and mediation of social and economic interests about the patrimonial possessions pursuing Simmel's notion of conflict described in a positive way in order to illustrate her function as a producer of social cohesion. In heritage city's financial public and private interests encourage the development of cultural activities, such as the cultural tourism and the improvement of historical buildings. Therefore Simmel's notion of conflict allows us to understand the effects of the politics of development and improvement of the so-called "patrimonial" urban space. Indeed, new forms of power and transactional processes impact the local territorial areas through the valorization and de-valorization of urban space.

Keywords: conflict, social transaction, city, heritage, heritage forms.

1 Département de sociologie de l'Université de Valparaiso au Chili. 


\section{Introduction}

Espace et forme spatiale de la modernité, la ville constitue un objet interdisciplinaire par excellence auquel s'intéressent de nombreuses sciences humaines et sociales, notamment au travers de la problématique du conflit, à la fois coexistence et cohabitation territoriale des intérêts sociaux. La sociologie simmelienne, en particulier, permet de comprendre les différentes formes de socialisation, qui sont à l'origine de la transaction sociale. Il s'agit d'une théorie qui constitue un outil favorisant la compréhension des conflits d'intérêt et des mécanismes de médiations sociales entre différentes compositions socio-spatiales. À l'aide de la sociologie de Georg Simmel, deux axes d'analyse peuvent être développés concernant la morphologie de l'habitat : d'un côté, la ville comme une structure d'efficacité fonctionnelle et, de l'autre, la ville comme milieu socio-affectif. À travers ces deux axes, les quartiers établissent un rapport de territorialité avec l'espace urbain, basé sur le degré de signification que peuvent acquérir les rues et les places comme lieux de rencontre et lieux socio-affectives de partage.

Les concepts de conflit et de forme, chez Simmel, nous amènent à un corpus de réflexion utile pour comprendre le jeu des acteurs situés dans les conflits pour l'appropriation des biens dits patrimoniaux. D'abord, pour Simmel, le conflit, en tant forme de socialisation, a « une signification sociologique, puisqu'il suscite ou modifie des communautés d'intérêt, des regroupements en unités, des organisations : voilà un principe qui n'a jamais été contesté » (Simmel, 2003:19). En effet, l'habitat est une ressource sociale et un outil pour l'analyse de nouveaux processus transactionnels dans l'espace urbain patrimonial. Nous nous intéressons ici au conflit existant dans les villes patrimonialisées, plus précisément celui entre les quartiers centraux et les quartiers péricentraux à Strasbourg et à Valparaiso, deux villes qui sont inscrites au Patrimoine mondial de l'Humanité par l'UNESCO. À partir de deux textes de Georg Simmel, Le Conflit (2003) et Esthétique et sociologie (2006), nous mobilisons la morphologie urbaine et celle de l'habitat, comme un axe d'analyse pour comprendre les formes sociales dans un contexte de patrimonialisation.

Notre hypothèse est que le passage de la méconnaissance/dévalorisation à la reconnaissance/valorisation du patrimoine bâti produit une reconfiguration de la structure socio-spatiale des anciens quartiers péricentraux, dont la patrimonialisation modifie les formes de socialisation en déclenchant de nouveaux jeux d'acteurs et des expertises, en produisant une cohabitation conflictuelle entre la valeur matérielle et immatérielle du patrimoine bâti. Dans une première partie, nous examinons la notion de ville patrimonialisée sous l'œil de la sociologie simmelienne, en intégrant les études de cas enquêtés : La Krutenau et le quartier de la Gare, à Strasbourg, et le Cerro Alegre et le Cerro Cordillera, à Valparaiso (section 1). Ensuite, nous abordons le concept de conflit et les processus transactionnels derrière le patrimoine bâti, en articulant aussi les études de cas analysés (section 2). 
Cette étude se fonde sur les résultats de la thèse doctorale de l'auteur soutenue en 2012 à l'Université de Strasbourg. L'enquête de terrain réalisée a suivi une méthodologie qualitative inscrite dans une analyse comparative dans trois villes qui ont vécu des effets des processus de patrimonialisation à une échelle moyenne. Il s'agit d'analyser les logiques socioculturelles de la patrimonialisation péricentrale de trois villes, Bâle, Strasbourg et Valparaiso. Trois villes qui ont une attractivité historique reconnue à l'échelle internationale, nationale et régionale, et dont le patrimoine bâti est un point d'intérêt pour le marché culturel et économique depuis de nombreuses décennies. Le choix des terrains de recherche s'est fait à partir de trois critères de sélection : i) des villes qui représentent un intérêt patrimonial au niveau international, national et régional, ii) des villes qui ont une attractivité touristique et commerciale de longue date, iii) des villes qui ont expérimenté des opérations de revalorisation et de réhabilitation du patrimoine bâti dans des quartiers anciens péricentraux.

Dans cette contribution, nous avons retenu seulement les résultats sortants de ces dernières villes, Strasbourg et Valparaiso, et quatre quartiers enquêtés : La Krutenau et le quartier de la Gare, à Strasbourg, et Cerro Alegre et Cerro Cordillera, à Valparaiso. L'étude, réalisée entre septembre 2007 et juillet 2011, a combiné des observations participantes, des entretiens semi-structurés avec des personnes-ressources, des informateurs et des experts, ainsi que des questionnaires auprès d'un échantillon d'habitants des quartiers étudiés ( 45 habitants par quartier). La recherche menée inclut aussi le dépouillement des archives et de documents officiels par rapport aux données sur le développement socio-historique des quartiers réhabilités.

Avec ces deux études de cas, nous mettons en lumière un exemple précis de pays dit "du Nord », la France, et un exemple d'un pays dit «du Sud», le Chili — deux villes classées au titre du Patrimoine de l'Humanité UNESCO. Dans ce contexte, nous avons mis en place le paradigme méthodologique de la transaction sociale pour nous fournir des outils nécessaires afin de construire le modèle de l'enquête : «repérer les oppositions structurantes, donner un statut à la dimension matérielle, dégager des séquences, identifier des produits transactionnels, indiquer l'une ou l'autre inférence aboutissant à des effets de sommation » (Remy, $1998 \mathrm{a}, 31-32)$.

L'approche comparative de cette recherche a mis en place des instruments qualitatifs et quantitatifs similaires dans la collecte pour permettre de croiser les variables dans l'analyse micro-sociologique: exploration photographique, enquête ethnographique pour identifier les acteurs pertinents, grille d'entretien et questionnaires. Les terrains étudiés permettent de comparer les logiques socio-spatiales à l'œuvre dans deux contextes semblables et différents à la fois. Il s'agit d'une analyse comparative qui se déplace entre les particularismes singuliers et les généralités plurielles pour fixer des coordonnés entre les terrains de recherche et le chercheur comparatiste. L'étude se situe dans un cadre "inter-national» de recherche, c'est-à-dire les outils méthodologiques s'articulent entre une échelle macro, où les objets étudies sont situés, et micro, où ces mêmes objets sont observés (Maurice, 1989). 


\section{La ville patrimonialisée au prisme de la sociologie simmelienne}

Nous souhaitons développer une sociologie de la ville s'appuyant sur le concept de forme, car il nous permet de développer une approche enrichissant la problématique actuelle des conflits d'intérêts, en prenant le patrimoine comme élément d'analyse. Chez Simmel, le concept de forme se situe au milieu de la métropole comme produit matériel de la modernité, où se produisent des formes de sociabilité et des formes esthétiques précises. La ville patrimonialisée, comme construction sociale, se constitue d'une superposition de couches, des formes matérielles y coexistaient renvoyant aux époques antérieures. Ainsi, tant Strasbourg comme Valparaiso sont deux exemples de villes modernes au sein desquelles coexistent ces formes héritées du passé, à la fois industrielles et commerciales. C'est là où se développe un carrefour entre l'homogénéité valorisée et l'hétérogénéité dévalorisée de formes matérielles esthétiques, si on reste dans l'ordre du discours du classement UNESCO. Ainsi, par exemple, les traces du commerce du XIX ${ }^{\mathrm{e}}$ siècle et d'une architecture de style victorien à Valparaiso portent plus de valeur patrimoniale que les traces d'une architecture spontanée rurale.

La métropole, chez Simmel, est la « ville de la modernité » qui représente une rupture avec la « ville historique », même si elle cohabite avec des formes du passé. La métropole constitue une société urbaine à l'écart des petites villes ou des villages, car les compositions sociales et les dynamiques urbaines deviennent plus complexes dans la différenciation sociale et culturelle de l'espace urbain.

La ville, chez Simmel, est à la fois une forme esthétique et une modalité de structuration du social : «les actions réciproques qui s'y déroulent sont-elles constitutives de la ville comme forme esthétique. Elle est une œuvre collective muée par une dynamique commune d'auto-organisation» (Remy, 1995: 9). Il s'agit d'une forme esthétique par rapport aux formes matérielles qui sont plutôt asymétriques que symétriques, en suscitant plusieurs fonctionnalités dans la distribution de formes sociales en ville. Néanmoins, la notion de ville patrimonialisée acquiert une mise en valeur depuis une forme esthétique symétrique, ou plutôt homogène, comme une idée de ville historique qui répond à une époque précise de reconnaissance. À Strasbourg, par exemple, l'image qui a été plus reconnue par la Mairie et l'UNESCO était la forme urbaine esthétique du Moyen Âge concentrée dans l'« ellipse insulaire », même s'il existe une grande partie de la ville qui réponde à des traces de la Renaissance ou de l'époque impériale que l'on peut identifier dans les quartiers péricentraux, comme le quartier de la Place de la République (cf. Carte 1) 
Par conséquent, la configuration de la ville patrimonialisée se produit à partir de la mise en valeur de l'esthétique architecturale, mais aussi par un ordre du discours sur le patrimoine qui s'installe. Ainsi, une forme urbaine patrimoniale se constitue à partir d'une image historique reconnue, mais aussi par un discours structurant développé par une expertise savante qui valorise les biens culturels par rapport au critère de «valeur d'exception universelle», c'est-à-dire une valeur particulière d'authenticité qui contient les biens.

\subsection{Le patrimoine bâti : entre conflit et forme}

Carte 1 : Secteur sauvegardé et périmètre du classement UNESCO²

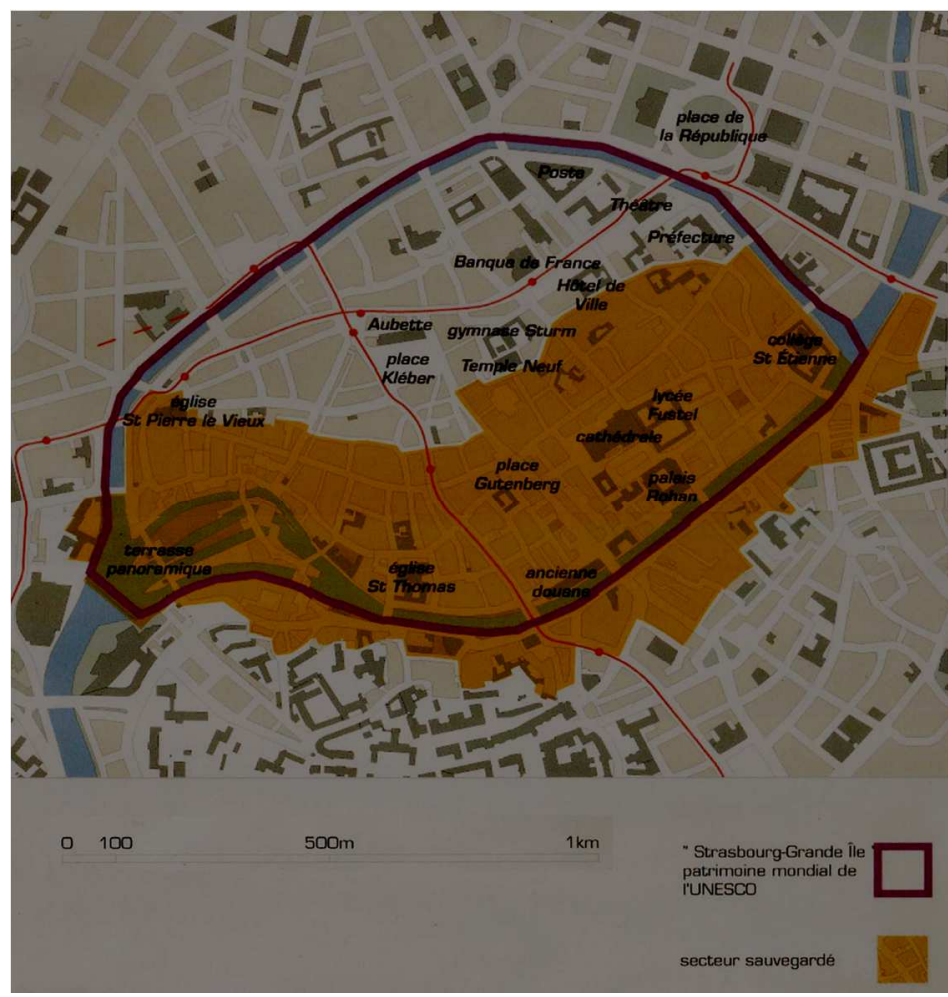

Dans notre analyse, nous abordons ce concept sous l'angle d'une valorisation des éléments architecturaux en tant que conteneurs des formes sociales passés et présents. Le patrimoine architectural, élément structuré et structurant de la mémoire et de l'identité locale, peut tant être approché par un regard plutôt rétrospectif que prospectif, où

2 Département de Développement Urbain (DDU).Programmation et conception urbaine. Système d'Information Géographique- Communauté Urbaine de Strasbourg (SIG-CUS), 2004. 
la connexion entre la forme urbaine patrimoniale et la vie sociale patrimoniale est à l'origine d'une nouvelle configuration des processus transactionnels. De plus, il inclut simultanément une dimension individuelle et collective, où la force du passé réside dans l'héritage transmis. Le patrimoine architectural reste parmi les différentes formes de patrimonialisation, d'un côté la valorisation endogène par les acteurs locaux et les habitants eux-mêmes ; de l'autre, celle émanant des acteurs exogènes, nationaux et internationaux, l'UNESCO par exemple.

Dans ce contexte, le conflit simmelien a une signification sociologique, «puisqu'il suscite ou modifie des communautés d'intérêt» (Simmel, 2003 : 19). Ainsi, les relations sociales des individus poursuivant des intérêts propres se soudent et deviennent une unité au sein de laquelle ces intérêts se réalisent. Il existe, en même temps, une communauté d'intérêts partagés où se retrouvent les individus qui appartiennent à différents cercles sociaux selon leurs expertises 3 . Au sein de ces cercles d'appartenance et d'expertises cohabitent les conflits internes au moment de préparer un dossier pour classer un quartier ancien, par exemple, ou déclencher une opération de rénovation d'un vieil immeuble.

Le conflit est une forme sociale composée d'oppositions, de différences et d'intérêts divers. Comme l'affirme Simmel : «C'est précisément en s'en différenciant que le conflit désigne le moment positif qui tisse avec son caractère de négociation une unité qu'on ne peut défaire que conceptuellement, mais pas en fait» (Simmel, 2003: 20). Chez cet auteur, le conflit est une forme de sociabilité qui agit sur des forces opposées et réciproques, à travers d'un processus d'antagonisme, ou plutôt conflictuel, comme c'est le cas avec la mise en valeur des funiculaires à Valparaiso (Soto, 2014). Concernant ce conflit, l'antagonisme constitue une force enrichissante pour les enjeux sociaux, dont la négociation est naturelle et le consensus sans négociation serait une forme anti-naturelle de négociation. La coexistence des formes diverses constitue un élément de liaison dans l'hétérogénéité et les différences esthétiques de la ville patrimoniale. Pour Simmel, la symétrie « veut dire, esthétiquement, que l'élément singulier dépend de son interaction avec toutes les autres, mais, en même temps, que la sphère ainsi désignée est fermée sur elle-même. Les formations asymétriques au contraire accordent, avec un droit plus individualisé pour chaque élément, une plus grande marge pour des relations qui s'étendent librement dans l'espace » (Simmel, 2006:135). De cette manière, on peut se poser la question si le lien social est fort ou non à l'intérieur d'une communauté donnée qui habite un immeuble ancien, où la coexistence des formes matérielles valorisées et dévalorisées est un élément contribuant aux conflits internes de la vie sociale.

3 L'expertise est «l'ensemble des positions sociales que produit la tension entre l'exercice professionnellement contrôlé et l'exercice auto-proclamé d'une compétence, en réponse à des situations confuses appelant une décision d'attribution ou de validation» (Trépos, 1996 : 203). Trépos fait par exemple la distinction «l'expert-aménageur » et «l'usager-expert », car la circulation des compétences et des atouts n'est pas la même dans la connaissance pour répondre aux défis de la patrimonialisation et de la revalorisation d'un quartier ancien. 
Dans ce contexte, l'habitat est une ressource sociale relevant de l'analyse des conflits et, surtout, de nouveaux processus transactionnels dans l'espace urbain bâti, illustré par les différences entre les quartiers centraux et péricentraux à Strasbourg et à Valparaiso. Ainsi, les transactions sociales permettent de comprendre les différentes formes de socialisation à partir de la mise en place d'outils de médiation ou de négociation dans un terrain conflictuel, comme les processus de valorisation ou dévalorisation du patrimoine bâti. «L'architectonique spatiale est une médiation qui agit comme révélateur et modifie le champ de potentialités» (Remy, 1999:178).

En fait, la ville est un lieu d'échanges entre les différents acteurs sociaux, politiques et économiques, autour des services et des biens, en donnant origine à des nouvelles dynamiques de peuplement urbain à partir de la valorisation d'une forme urbaine historique, unique et confortable. Ce processus agit sur deux formes de dévalorisation et valorisation de l'image patrimoniale, à savoir «l'une concerne le bâti ancien dévalorisé et réhabilité plutôt de façon individuelle et l'autre relève de la promotion immobilière sur des terrains en cours de valorisation foncière» (Bidou-Zachariasen, 2003: 14). L'image valorisée permet de comprendre certains processus de la vie sociale patrimoniale qui concerne des quartiers anciens améliorés et aménagés, à condition que ces quartiers soient centraux ou péricentraux, comme nous allons essayer de le démontrer dans les pages suivantes.

\subsection{Le patrimoine architectural : espace de conflit entre la centralité et la péricentralité}

Le patrimoine est un concept polysémique. Dans une dimension économique, on parle de patrimoine et de capital, c'est-à-dire de l'héritage économique au sens de la transmission de la fortune, d'un côté, et de l'accumulation de biens à marchander dans le marché financier et commercial, de l'autre. Cependant, le patrimoine a un sens culturel et historique lié à l'histoire d'un pays et à ses monuments. Néanmoins, l'association de la notion de patrimoine à la propriété comme signe de richesse peut poser problème. Le patrimoine bâti représente l'accumulation des biens et des propriétés, des archives et des documents, des images et des données, « il devient une valeur a priori, il se détermine comme origine même du lien entre valeur économique et valeur morale » (Jeudy, 1990 : 1). Le patrimoine architectural représente donc un héritage et un transmetteur. Par conséquent, la question est de savoir quel est l'héritier qui garde le patrimoine bâti et comment il faut le transmettre.

Tout d'abord, on peut relever une distinction entre l'ancien et l'historique dans les villes avec une connotation patrimoniale. Les quartiers anciens sont des quartiers qui portent des traces du passé. Les quartiers reconnus comme historiques sont des quartiers déjà touchés par des opérations de revalorisation dans la logique de la réhabilitation, de la récupération et du « recyclage» de la structure bâtie, ou plutôt de la matérialité (Coing, 1966; Bourdin, 1984). L'ancienneté correspond à des traces du passé 
dévalorisées et au fait de mettre en valeur celles-ci en les inscrivant comme marques historiques dans une opération de politique patrimoniale.

Comme l'ont souligné Elias et Scotson (2001 [1965]), dans un quartier, l'ancienneté est un facteur puissant de légitimité : les habitants de longue date (established) ont plus de légitimité à parler au nom du quartier que les nouveaux venus (outsiders). Il en va de même pour les monuments et les immeubles : plus ils sont anciens plus ils sont considérés comme «chargés d'histoire» et moins leur légitimité historique est contestable. Mais cette dernière est insuffisante pour les mettre à l'abri des menaces de destruction dans le cadre de la spéculation immobilière.

Le quartier est une unité socio-spatiale proche du centre-ville avec une autonomie et homogénéité des formes et fonctions. Il constitue une sorte de culture locale avec des particularités de formes, de rituels ou de marquages de l'espace d'appartenance. L'analyse microsociale d'un quartier concerne l'étude de la spatialisation de sa vie sociale. Les pratiques sociales et culturelles s'expriment, même si la réalité reste conflictuelle entre les différents acteurs (Blanc, 2006). La morphologie du quartier renvoie à sa forme et à son image physique. La place du quartier dans l'espace urbain est un élément important, en particulier sa proximité (ou sa distance) par rapport au centre. Sous des appellations fluctuantes et imprécises, on distingue les quartiers centraux des quartiers périphériques, avec des distinctions internes (faubourgs, banlieues, etc.). On parle notamment de première et de deuxième couronnes lorsque la croissance urbaine et le développement de l'urbanisation font que des quartiers autrefois périphériques sont successivement intégrés dans un centre élargi. Ces quartiers deviennent des quartiers péricentraux.

Dans la plupart des cas «les quartiers péricentraux correspondent aux anciens faubourgs et aux banlieues ouvrières, où s'entremêlent des vastes emprises industrielles désaffectées et des poches d'habitat ancien dégradé » (Linossier et al., 2004 : 24). Ceuxci correspondent à la première couronne de la ville, même si, à Valparaiso, il est difficile de faire la différence entre une colline et un quartier, car les deux s'imbriquent mutuellement dans la morphologie spatiale et sociale de la ville. On peut également identifier une coexistence de formes architecturales entre le centre-ville et les quartiers péricentraux de la première couronne, qui constituent des liaisons entre les étapes du développement urbain de la ville. Le centre des villes patrimonialisées a une valeur historique. Celui-là dispose d'équipements culturels et de loisirs ainsi que de nouveaux lieux de consommation qui attirent les habitants comme les touristes. Ainsi se constitue la figure spatiale d'une nouvelle centralité grâce aux attractions touristiques au service du patrimoine (Vermeersch, 2003). Les relations socio-spatiales s'articulent autour de l'accessibilité aux biens et aux services du centre-ville: les habitants font un arbitrage entre la proximité du centre et la qualité de l'habitat. Les quartiers historiques péricentraux offrent un compromis acceptable pour les classes moyennes aisées : près du centre, des logements spacieux et confortables sont à un prix abordable. 
Les quartiers péricentraux bénéficient de certains atouts pour devenir des espaces urbains attractifs : proches du centre-ville et, dans certains cas, des immeubles anciens à réhabiliter ou à restaurer pour de nouveaux usages résidentiels ou commerciaux. Ces avantages peuvent se résumer par deux mots : confort et nostalgie. «Le goût pour le patrimoine est une forme de consommation, mais à la fois une production des particularités culturelles dans un contexte global» (Le Goff, 1988 : 59). Ces quartiers représentent de notables attractivités pour les investissements privés et les promoteurs immobiliers, dont le goût pour l'ancien structure les marchandises du patrimoine à consommer.

La différence entre centralité et péricentralité réside dans un processus de croissance urbaine comme l'a bien démontré Ernest Burgess par rapport à la structure sociale et à la mobilité urbaine (Burgess, 1967 [1925]). La ville dispose d'un centre d'affaires entouré par une zone d'industries légères, au-delà de laquelle il y a une «zone de transition» correspondant aux quartiers péricentraux qui l'entoure. Les cercles concentriques situés plus loin, qui forment la «zone des banlieusards», correspondent aux quartiers périphériques ${ }^{4}$. La centralité et la péricentralité concernent des distinctions spatiales par rapport aux différentes étapes du développement d'une ville. Les quartiers centraux sont le point de départ dans sa planification ou sa croissance ; c'est un processus tantôt planifié, tantôt spontané. Les quartiers péricentraux marquent une étape postérieure dans le développement urbain, l'arrivée de nouvelles populations et l'installation de nouvelles activités économiques déclenchant la naissance de nouveaux quartiers.

Dans certaines villes, la péricentralité porte une image dévalorisée par rapport à la qualité de son habitat, sa sécurité, ses services et ses équipements. Cependant, les quartiers péricentraux ont des structures bâties susceptibles d'être récupérées et mises en valeur, même s'il s'agit d'une usine ou d'un ensemble d'habitats ouvriers. En fait, ils sont concernés par les opérations de rénovation et réhabilitation. Ils portent les représentations sociales d'un patrimoine bâti qui concerne généralement une multiplicité de styles architecturaux et d'usages qui gardent des traces d'un passé « digne » à mettre en valeur par les acteurs de la patrimonialisation.

Les quartiers anciens péricentraux sont à proximité du centre, tout en étant distincts de lui. Ils sont peu à peu intégrés dans un centre élargi. En fait, ces quartiers se «muséifient» et deviennent des extensions du centre historique, permettant de lutter contre la saturation de l'hyper-centre et de mieux répartir les flux touristiques, en structurant une sorte de «patrimonialisation péricentrale». Ensuite, l'amélioration de l'habitat rend le quartier très attractif pour une population plus aisé. La spéculation est

4 Le schéma de Burgess se structure avec des cercles concentriques, un modèle qui s'inspire de Chicago. Cependant d'autres villes ont suivi des modèles de planification différents, en fonction de la morphologie physique de la ville, comme Valparaiso avec ses 42 collines, ou de processus culturels et politiques, comme, par exemple, les villes coloniales fondées par les espagnols en Amérique Latine à partir d'un plan en damier. 
à l'origine d'un processus spécifique d'embourgeoisement urbain, la gentrification, qui met en valeur le caractère "populaire » du quartier, tout en expulsant les anciens résidents (Authier, Bidou, 2008).

La différence entre les quartiers historiques péricentraux tient à leur localisation: dans le cas de Strasbourg, la coupure est évidente entre la Grande-île et les quartiers voisins délimités par le fleuve qui entoure le centre historique, l’lll, et les remparts. C'est l'opposition entre intra - et extra-muros, une distinction moins visible à Valparaiso entre les quartiers centraux et péricentraux 5 . Dans ces processus, les représentations patrimoniales, qui construisent les individus, sont organisées par rapport à deux couples de tension. D'un côté le patrimoine et l'« ancienneté reconnue» du quartier et de l'autre, l'axe organisateur et l'image principale du quartier.

Le problème majeur des quartiers péricentraux, c'est de se distinguer les uns des autres, tout en affirmant leur histoire et leur identité propre. En paraphrasant Axel Honneth (2002 [1992]), ils entrent dans «une lutte pour la reconnaissance». Il s'agit de faire admettre des formes plurielles de légitimité, niées par le modèle monolithique dominant. Les immeubles industriels peuvent devenir les témoins des conditions de vie et de travail d'une époque antérieure. Ce qui peut aboutir à des formes spécifiques de tourisme culturel et de tension, comme nous allons le voir dans le paragraphe suivant.

\subsubsection{Le patrimoine comme forme de socialisation conflictuelle à Strasbourg}

Strasbourg est une ville frontalière entre la France et l'Allemagne. Elle a été fondée par l'occupant romain comme place militaire sous le nom d'Argentoratum en 12 avant J.C.. Ensuite, elle a évolué pour devenir un bourg de commerçants et d'artisans au Moyen Âge, avant d'aboutir à un carrefour au cœur de l'Europe pour les échanges culturels, politiques, économiques et religieux avec la présence des trois cultes principaux : catholique, protestante et juive.

C'est grâce à cette situation frontalière que cette ville a construit son identité. Le canal dit des Faux Remparts délimite le centre de la ville, appelé «ellipse insulaire» ou «Grand-Île ». C'est précisément ce périmètre qui a été nommé au titre du Patrimoine mondial de l'Humanité en 1988: la cathédrale comme réalisation artistique unique, ses qualités en tant que vecteur de l'art gothique en Europe, et la Grande-Île, ensemble urbain caractéristique de l'Europe moyenne et ensemble exceptionnel d'architecture typique (cf. Fig. 1).

5 Les villes européennes classées par l'UNESCO sont souvent d'anciennes villes fortifiées, avec une coupure nette entre le dedans et le dehors de la ville (faubourg, banlieue). Mais cette distinction ne coïncide pas avec celle des quartiers bourgeois et populaires. À Valparaiso, comme dans la plupart des villes en Amérique latine, il n'y a jamais eu des fortifications et la frontière entre les quartiers péricentraux et le centre est parfois assez imprécise. 
Dans l'ellipse insulaire, les quartiers du centre-ville ont gardé une morphologie de ville médiévale avec des toponymes. Citons l'exemple des rues portant la mémoire de leurs anciennes fonctionnalités urbaines et leurs caractères résidentiels : la place du Vieux-Marché-aux-Poissons ou la rue des Juifs. Les quartiers péricentraux de la première couronne ont acquis une physionomie industrielle : c'est le cas des usines de gaz et d'électricité du quartier de la Gare et de la Manufacture des Tabacs du quartier de la Krutenau.

Fig. 1 : Section du secteur sauvegardé PSMV et classé par l'UNESCO

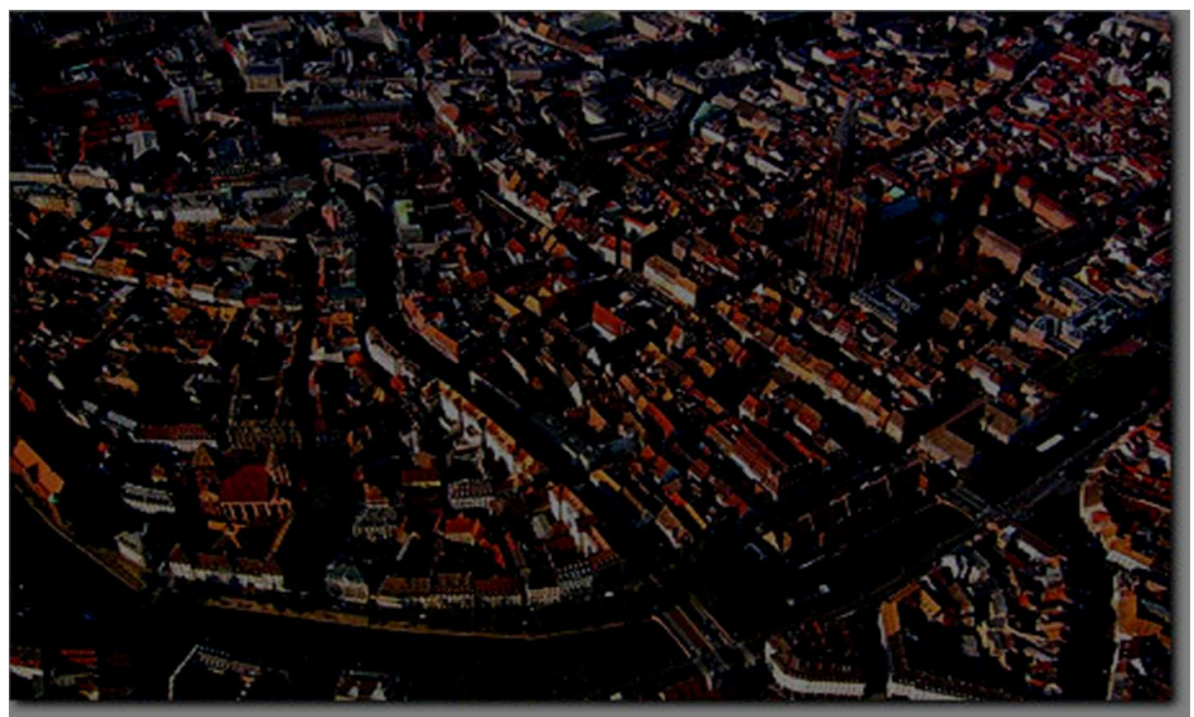

Source: site internet de la Ville de Strasbourg, 2010

Le quartier de la Gare représente l'évolution urbaine entre l'ancien centre-ville, les anciennes routes de connexion avec la ville extra-muros et les lotissements industriels du XIX ${ }^{\mathrm{e}}$ siècle. À la Gare, le secteur le plus ancien constitue la trame des îlots Faubourg National, et de certaines rues (rue de la Course, rue Déserte, rue du Maire Kuss, rue Kageneck et rue Faubourg de Saverne). C'est un périmètre privilégié pour les opérations de réhabilitation et d'intervention dans un patrimoine architectural de pierre taillée. L'histoire du paysage urbain y rappelle les grandes étapes de la formation du quartier, à partir duquel on peut arriver à une meilleure compréhension de la morphologie du quartier autour d'un patrimoine industriel et «populaire». Particulièrement dans le sous-quartier Faubourg de Saverne, on voit un espace qui concentre non seulement des activités résidentielles, commerciales et industrielles, mais aussi un espace stratégique pour la mise en valeur du patrimoine architectural par sa proximité du centre-ville et de la Gare Centrale.

En plus, le quartier de la Krutenau se trouve, quant à lui, dans la continuation immédiate du centre-ville dans la direction sud-est. Il a été qualifié de banlieue, de faubourg 
et de quartier dans le développement urbanistique de Strasbourg. Grâce à ces nombreux canaux, ce quartier a pu accueillir des activités maraîchères. "Cité des eaux, bâtie sur un véritable marécage et peuplée de bateliers, mariniers et pêcheurs, la Krutenau fut très longtemps un quartier modeste, aux lacis de ruelles étroites et humides, bordées de maisons vétustes et basses, très souvent à colombage » (Fossel, Coltat, 1983: 3). L'implantation de casernes militaires au XVIII siècle, puis d'une manufacture de tabacs au XIX ${ }^{e}$ siècle a également fait évoluer la population du quartier où les ouvriers et les soldats étaient prédominants. La Krutenau présente un patrimoine architectural notable qui correspond aux constructions à colombage. En raison de sa proximité du centre-ville et de ses différentes zones d'attractivité, ce quartier a été l'un des premiers à être ciblé par les mesures politiques de la lutte contre l'insalubrité de l'habitat, avant que ne soit engagée sa rénovation par la politique de renouvellement urbain vers la fin des années 1970.

En fait, la Krutenau représente un quartier qui est marqué par différentes périodes dans la composition sociale de sa population. En 1975, les ouvriers représentaient la moitié des habitants du quartier, mais en 1982 ils n'en constituent qu'un tires (INSEE, 1975; INSEE, 1982). Cette évolution représente un atout pour la spéculation immobilière des années soixante-dix, du fait des éléments ciblés dans la stratégie de la politique foncière à la Krutenau, par exemple la proximité du centre-ville et le patrimoine architectural renouvelé.

Au cours des années 1960 et 1970, la mise en place des opérations de rénovation-démolition établies par le décret-loi du 31 décembre 1958 a conduit à la destruction d'une grande partie des anciens quartiers. Sans devenir des secteurs sauvegardés, certains quartiers, comme la Krutenau et la Gare, ont survécu et ont été réhabilités parce qu'ils étaient proches du secteur sauvegardé. Ainsi, le rapport entre la sauvegarde patrimoniale de quartiers centraux et la réhabilitation de l'habitat ancien de quartiers péricentraux met en valeur un patrimoine bâti pour sa proximité au centre-ville.

Les secteurs sauvegardés sont porteurs d'une légitimité de l'histoire urbaine d'une ville. Le classement d'un quartier ancien comme quartier historique est donc un acte politique qui revalorise le quartier en déclenchant la valeur marchande associée aux biens dits patrimoniaux, soit dans les façades restaurées ou dans les anciennes maisons bourgeoises dégradées qui ont été transformées, par exemple, en locaux commerciaux de luxe. «L'émergence progressive des pouvoirs locaux va s'accompagner d'une prise de conscience des mémoires locales » (Serfaty-Garzon, 1992: 374). Dans ce contexte on peut se poser la question de savoir si la sauvegarde d'un site peut déclencher une nouvelle appropriation?

La patrimonialisation des quartiers anciens centraux et péricentraux s'appuie sur des dispositifs développés dans les discours et les actions concrètes de la politique patrimoniale, soit par la réhabilitation aux échelons national, régional ou local de la politique publique, soit par le classement par l'expertise internationale du patrimoine. D’autres procédures mettent en valeur l'habitat ancien dévalorisé et vétuste qui se 
trouve parfois situé à l'intérieur ou à proximité des secteurs du Plan de sauvegarde et de mise en valeur (PSMV). C'est le cas des Opérations programmées pour l'amélioration de l'habitat (OPAH). Dans le cas de Strasbourg, certaines OPAH ont su réhabiliter des quartiers anciens dégradés, en permettant la revalorisation d'un patrimoine dévalorisé et parfois méconnu, menacé de disparition, voire de démolition. C’est le cas des OPAH qui ont été réalisées à la Krutenau entre 1977-1980 et dans le quartier de la Gare entre 1988-1992. La seule OPAH qui s'est développée dans la Grande-Île concerne la Grand'Rue ${ }^{6}$, une opération qui a commencé au milieu des années 1980 et qui est arrivée à son terme en 1988, c'est-à-dire dans l'année du classement de la Grande-Île par l'UNESCO.

L'OPAH n'a pas pour objectif prioritaire de revaloriser le patrimoine bâti, car elle est une procédure destinée à produire des logements dans le contexte de la réhabilitation de l'habitat ancien et vétuste, voire insalubre. Cependant, l'amélioration de l'habitat ancien déclenche une revalorisation du tissu bâti dans les quartiers affectés et dans les activités commerciales. Ensuite, un classement UNESCO peut produire un effet d'accélérateur de la revalorisation et de la gentrification du quartier. Une OPAH a pour but de préserver et d'améliorer le patrimoine immobilier existant, en définissant de normes pour la réhabilitation des logements ou l'amélioration de l'habitat à travers l'angle de la cohésion territoriale et de la réduction des problèmes sociaux comme celui de la pauvreté. Les principaux objectifs considérés sont les suivants : la production de logements et l'amélioration du confort des logements, tout en intégrant les ménages aux ressources modestes, ainsi que les travaux d'amélioration de l'habitat par une politique d'aménagement. Les OPAH sont conçues comme des opérations devant aborder l'ensemble des problèmes que peut rencontrer un quartier (logement, équipements, commerces, écoles...). Le but consiste à intervenir dans des zones d'habitat vétuste et dévalorisé, tant en ville comme en milieu rural. C'est ainsi que le patrimoine bâti des quartiers anciens centraux et péricentraux est revalorisé par ricochet, en produisant une image plus attirante pour ceux qui veulent vivre dans un appartement ancien, mais avec le confort d'un appartement moderne et proche du centre-ville.

Dans le cas de Strasbourg, les procédures OPAH répondent à une politique de réhabilitation de l'habitat ancien à l'échelle nationale qui s'organise autour de quatre axes : i) maintenir la population de ces quartiers en améliorant le confort des logements et l'agrément du cadre de vie ; ii) attirer une population nouvelle afin d'éviter l'anémie de ces quartiers ; iii) sauvegarder le domaine bâti dans un état satisfaisant de maintenance d'aspect et de confort ; et iv) satisfaire des besoins sociaux qui jusqu'alors n'ont été que très partiellement et imparfaitement pris en compte (Lévy, 1990). Les deux premiers axes sont contradictoires, car maintenir la population et en même temps en attirer une

6 La Grand'Rue est un ancien axe de sortie et d'entrée au centre-ville, a de l'Église Saint-Pierre le Vieux jusqu'à la rue de la Division Leclerc, non loin de la Place Gutenberg. C'est une rue à l'intérieur du PSMV, dont la réhabilitation a été suivie de façon vigilante par l'association des Amis du Vieux Strasbourg. 
nouvelle produit un processus de rupture sociale à l'intérieur des quartiers réhabilités, ce qui prend la forme de relogements spontanés ou forcés de l'ancienne population résidante. C'est ainsi qu'à la Krutenau et à la Gare, on constate des changements dans leurs structures socio-spatiales, où les anciens résidents ont laissé la place à une nouvelle population plus aisée qui a acheté des immeubles pour les louer aux étudiants (ADEUS, 2002a et ADEUS, 2002b).

\subsubsection{Le patrimoine comme forme de socialisation conflictuelle à Valparaiso}

Valparaiso est le principal port du Chili, un port mythique qui fait cohabiter la plaine et les cerros (collines): Valparaiso est «la ville aux quarante-quatre collines» (Sáez, 2001). Cette Ville-Port marquée par une morphologie spatiale dans la forme d'un amphithêâtre tend à confondre les collines avec les quartiers et les ravins (cf. Fig. 2). Cette singularité naturelle et culturelle représente la condition patrimoniale de Valparaiso structurée à partir de l'activité portuaire. Dans cette morphologie, on peut identifier deux collines, qui en raison de leur condition péricentrale, jouent un rôle au premier plan, le Cerro Alegre et le Cerro Cordillera. Cela ne s'explique pas seulement par leur topographie particulière, mais aussi par les activités commerciales, financières et sociales développées par les différents groupes sociaux qui se sont établis à l’ombre de la Ville-Port. 


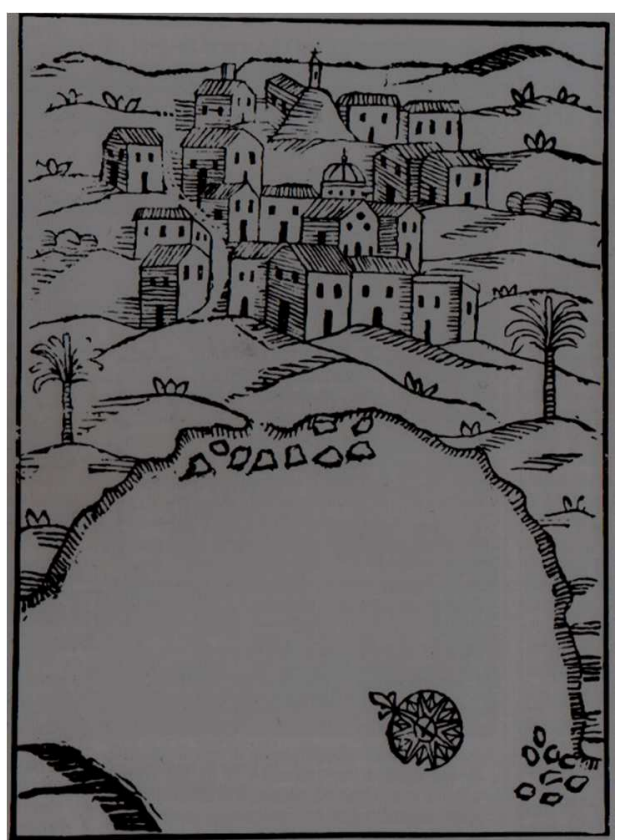

Fig.2. Première représentation graphique de Valparaiso par Alonso de Ovalle en 1646 (in Waisberg, 1992)

À la fin du XIX ${ }^{\mathrm{e}}$ siècle, la structure urbaine de Valparaiso est caractérisée par la présence de cinq secteurs clairement définis : le port où se situent les activités commerciales ; les collines Cordillera, Alegre et Concepción qui correspondent aux principaux lieux de résidence de l'archéo-bourgeoisie ; l’Almendral, quartier résidentiel et commercial situé dans la plaine ; le Cerro Barón, un autre quartier résidentiel, et finalement, Playa Ancha, secteurs résidentiels pour les ouvriers et pour les classes moyennes émergentes (Castillo, 2005). C'est dans sa morphologie que les quartiers ont évolué vers deux centres d'activité : le port, proche au Cerro Cordillera, et le quartier financier, proche au Cerro Alegre et Cerro Concepción.

À Valparaiso, on peut identifier différents types de patrimoine bâti, de constructions qui répondent aux différentes périodes de l'histoire de la Ville-Port. Par exemple, certaines constructions que l'on peut trouver au Cerro Cordillera répondent à un style rural de construction, dont les maisons ont été bâties en torchis pour les migrants de la campagne qui sont venus tout au long du $\mathrm{XIX}^{\mathrm{e}}$ siècle et au début du $\mathrm{XX}^{\mathrm{e}}$ siècle à la recherche d'une meilleure qualité de vie. Dans cette même colline, on peut identifier aussi des traces de constructions populaires, les « cités », qui ressemblent au modèle de 
phalanstère7. C'est le cas du bâtiment Población Obrera de La Unión (Soto, 2011). À la fin du XIX siècle, elles ont répondu au besoin d'habitat dans les secteurs populaires, une conséquence de l'exode rural. Au recensement de 1885, Valparaiso comptait 430 cités, avec des ménages de 5 à 10 personnes par logement.

Par ailleurs, la présence de riches immigrants anglais, américains et allemands au début du XIXe siècle, attirés par les activités commerciales et industrielles, a laissé des traces dans une architecture Art nouveau et néo-victorienne qu'on peut identifier aux Cerro Alegre et Cerro Concepción. Il y a avait, par exemple, en 1824 entre 1000 et 3000 Anglais installés à Valparaiso, dirigeant le commerce, la navigation et les affaires en général.

L'urbanisation du Cerro Alegre a débuté en 1820, à l'initiative de riches entrepreneurs anglais. En fait, ce quartier était le principal lieu de résidence des riches étrangers qui ont créé la première banque de Valparaiso en 1845 et la première Chambre de Commerce du Chili en 1917. Le Cerro Alegre est resté le quartier de prédilection de la bourgeoisie traditionnelle, à proximité du quartier financier de la ville. Cette colline se caractérise par un patrimoine bâti qui reflète la présence d'une immigration économiquement puissante qui a contribué à la croissance commerciale et économique de la ville au XIX ${ }^{\mathrm{e}}$ siècle.

Le Cerro Cordillera est proche de l'ancien centre commercial de Valparaiso. Il reste dans la zone valorisée, mais à la limite d'une zone dévalorisée et sans protection légale face à l'action des agents immobiliers et des promoteurs-investisseurs. Cette colline est passée d'une dévalorisation caractérisée par une population modeste aux faibles revenus à une revalorisation qui attire les investissements immobiliers et les touristes. Cette colline était stigmatisée dans les anciens itinéraires touristiques de la ville comme un lieu dangereux à éviter. Par contre, après le classement patrimonial de la ville, le Cerro Cordillera est devenu un lieu attractif pour les investisseurs immobiliers, surtout à proximité immédiate du centre patrimonial.

Depuis le classement d'une partie de la ville par l'UNESCO en 2003, des processus de patrimonialisation produisent de nouvelles formes de mise en valeur et d'appropriation de l'espace. Le dossier de candidature au Patrimoine mondial de l'Humanité avait pour principal objectif de développer un tourisme culturel, national et international, en donnant de Valparaiso l'image d'une "ville portuaire historique ». Cette candidature a été retenue selon le critère de "valeur universelle», comme "témoignage unique, ou du moins exceptionnel, sur la tradition culturelle ou sur une civilisation vivante ou disparue ${ }^{8}$.

\footnotetext{
7 Selon le règlement de 1899, les cités sont : « une propriété destinée à la location aux ouvriers de chambre ou de logements; une cité a plusieurs unités d'habitation ou sections du bâtiment qui ont une cour commune » (Revista de Higiene, 1901, traduction de l'auteur).

8 UNESCO, Convention concernant la protection du patrimoine mondial, culturel et naturel, 27e session, Paris, 2 juillet 2003.
} 
Si nous pensons au processus de patrimonialisation qui est en train de vivre la VillePort de Valparaiso depuis 2003 et même avant, on peut constater qu'elle suppose d'une mise en valeur des fonctions urbaines traditionnelles concentrées dans le quartier financier et les collines des alentours (Cerro Alegre et Cerro Concepción) qui appellent au principe de l'homogénéité des formes architecturales du patrimoine bâti. En fait, c'est un processus qui met plus en valeur les quartiers centraux que péricentraux, dont «la légitimation sociale de la centralité implique ainsi sa légitimation économique» (Vermeersch, 2003 : 617), mettant à l'écart des formes architecturales hétérogènes qui répondent aux différentes périodes de développement de la ville, et que l'on peut trouver au Cerro Cordillera (cf. Carte 2).

Dans cette logique, le Ministère de l'habitat et l'urbanisme (MINVU) a créé la Subvention de réhabilitation patrimoniale (SRP) sous la forme d'une aide financière à la réhabilitation de $6000 €$, pour attirer des investisseurs qui veulent acheter des immeubles de « valeur patrimoniale » et les diviser en plusieurs appartements (deux au minimum). Cette subvention est destinée à récupérer les anciens immeubles construits avant le 31.07.1959 ayant une surface maximale de $140 \mathrm{~m}^{2}$. La SRP s'applique à des immeubles situés dans la Zone de Conservation Patrimoniale et dans la Zone Typique. Toutefois, cette subvention pour la réhabilitation ne finance que des travaux dans des immeubles qui ont une valeur maximale de $44000 €$ dans les quartiers défavorisés, et de $73000 €$ dans les quartiers les plus dynamiques. Dans le premier cas, on trouve le secteur de Cerro Cordillera, où la SRP accompagne le programme de Réhabilitation des espaces publics du MINVU qui concerne les places publiques, les rues et les trottoirs. Dans le second cas, on trouve le Cerro Alegre, où la SRP a dynamisé une partie de l'activité commerciale et touristique du secteur, comme les bars, les restaurants et les cafés.

9 Date de publication dans le Journal officiel du décret de loi (DFL) n² 2, 31.07.1959. 


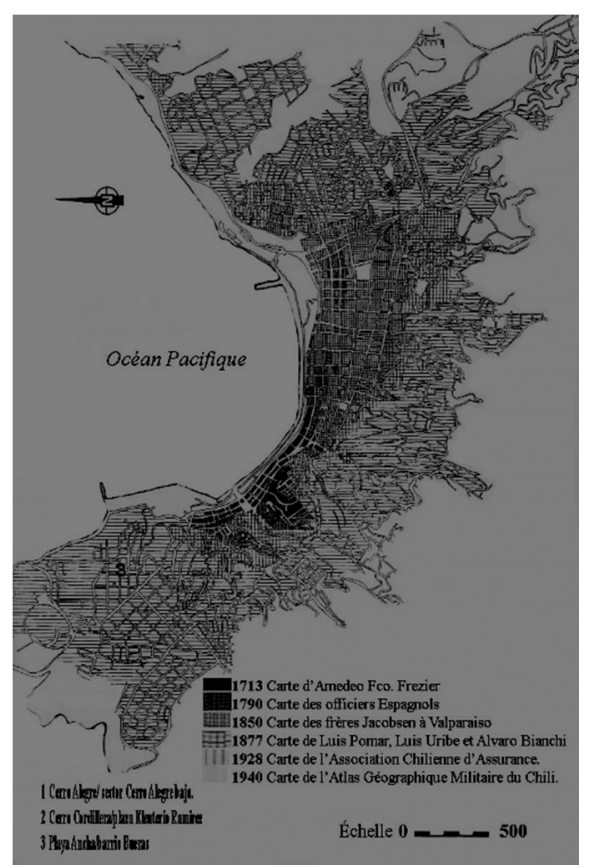

Carte 2 : Phases du développement urbain de Valparaiso entre 1713 et 1940 (in Benavides et al., 1994)

La réhabilitation patrimoniale a commencé par un exercice de reconnaissance des traces anciennes d'un bấtiment susceptible d'être récupéré en y ajoutant une valeur historique-patrimoniale. Cet exercice de distinction est développé à partir de l'avis des experts qui cherchent à récupérer des immeubles pour l'appropriation d'une minorité qui souhaite investir à Valparaiso, mais qui affecte le tissu social des quartiers anciens. Ainsi, même si la réhabilitation patrimoniale ne répond pas aux objectifs prévus en nombre d'immeubles réhabilités, les opérations développées dans le cadre de la SRP ont produit des re-localisations et des changements dans la composition sociale des collines proches de la zone du classement UNESCO qui intègre la zone de Conservation Patrimoniale (Trivelli et Nishimura, 2010).

La SRP s'illustre de manière claire comment la valeur matérielle d'un patrimoine bâti, conçue comme l'accélérateur du marché immobilier, dépasse la valeur immatérielle. Le MINVU oppose une logique économique à une logique sociale. La politique publique du patrimoine aborde la réhabilitation comme un outil essentiel dans la restructuration de nouveaux usages, mais sans les harmoniser avec les trois valeurs qui cohabitent à Valparaiso : la valeur urbaine, la valeur architecturale-historique et la valeur sociale. 
À partir des concepts de centralité la forme simmelienne, en tenant en compte les différences entre les quartiers centraux et péricentraux, on peut comprendre les différents niveaux de mise en valeur du patrimoine autour des formes valorisées et dévalorisées, entre une cité située au Cerro Cordillera et un immeuble style Art nouveau placé au Cerro Alegre. Au centre-ville, on peut voir « une centralité pratique vécue, qui se lit dans l'espace par l'animation des rues, la diversité des fonctions, et cette "effervescence sociale » qui lui confère son atmosphère caractéristique et sa reconnaissance comme l'espace commun, le lieu de rencontre des habitants de l'agglomération» (Vermeersch, 2003: 610). À Valparaiso, on peut vérifier la présence des critères économiques qui orchestrent les objectifs des instruments, comme le SRP, pour réhabiliter ou renouveler tel ou tel immeuble. Le patrimoine bâti joue donc un rôle important à partir des intérêts du marché pour comprendre les dynamiques sociales qui accompagnent un processus de patrimonialisation, comme celle de la gentrification et/ou de la muséification.

\section{Le conflit dans les villes patrimonialisées : le sens des transactions sociales}

Comme nous venons de constater, à partir du concept de forme simmelienne nous pouvons aborder le patrimoine bâti comme un mode de structuration du social, dont la bâti n'est pas un concept statique et fermé. Ainsi le patrimoine bâti, et surtout dans le contexte de villes patrimonialisées, devient un concept structurant relatif aux médiations et négociations, qui aide à comprendre la dynamique sociale existant entre les forces opposées dans un milieu conflictuel.

Le patrimoine bâti est un bien patrimonial qui a deux valeurs : une valeur matérielle et une valeur immatérielle. La matérialité correspond aux objets et biens, en articulant la dimension physique avec la rentabilité du bien; et l'immatérialité correspond aux représentations sociales qui gardent les objets et les biens, en les rattachant aux valeurs sociales et aux usages. Dans un processus de patrimonialisation, les quartiers anciens deviennent des espaces de conflit, car, pour les élus et les promoteurs-investisseurs, le patrimoine matériel est plus rentable que le patrimoine immatériel. En revanche, pour les experts, les habitants et les associations du quartier, le patrimoine représente d'abord une valeur symbolique et sociale multiple. Ainsi, l'opposition entre la conservation d'un vieil immeuble résidentiel au titre de bien patrimonial, pour un nouvel usage commercial, et la reconnaissance patrimoniale par ses habitants, est déjà une situation concrète conflictuelle entre la matérialité et l'immatérialité du bati ancien. Dans ce contexte, le conflit est une forme sociale par opposition aux couples de tensions qui se sont structurées à partir d'intéréts divers. En effet, le conflit est une des formes de socialisation les plus actives, impossibles à réduire à peu d'éléments, ce qui permet d'intégrer d'autres légitimités dans la patrimonialisation comme les expertises savantes et des expertises profanes des habitants. 
La patrimonialisation peut être abordée par les normes imposées non seulement par l'expertise internationale, notamment l'UNESCO et l'ICOMOS, mais également par la politique patrimoniale locale. Or, le concept de «diade», ou couples social, peut être élargi à une triade dont la troisième forme sociale, à savoir la mobilisation de citoyens, prend sa place dans l'analyse sociale de la patrimonialisation. Néanmoins, elle ne précise pas les types de conflits et les solutions provisoires existantes dans une négociation pour le nouvel usage d'un immeuble ancien.

Une telle dynamique sociale nous amène au terrain des transactions sociales. Ainsi, grâce à la triade, il est possible d'intégrer, par exemple, les avis des habitants par rapport à leur quartier. La transaction sociale reprend les valeurs telles que la légitimité, la reconnaissance, la solidarité, la confiance, la coopération, etc., pour faire des analyses qui aident à mieux comprendre le processus de patrimonialisation. «Les manières de faire ne désignent pas seulement des activités qu'une théorie se donnerait pour objets. Elles organisent aussi sa construction» (De Certeau, 2005: 119). Ainsi, la transaction sociale en tant que paradigme méthodologique «heuristique» (Blanc, 2009) intègre les formes actuelles de pouvoir, de négociation et de compromis, en permettant d'examiner la patrimonialisation dans les conflits d'usages et les représentations du patrimoine bati. En fait, la mise en place d'un processus transactionnel révèle les pratiques de co-présence dans les conflits patrimoniaux, dont les acteurs concernés sont en même temps proches et distants, en appelant à un processus de négociation en permanence pour satisfaire les acteurs d'une part et de l'autre.

\subsection{Les processus transactionnels derrière le patrimoine bâti}

La transaction sociale incorpore les notions de sens commun comme les négociations, les échanges, les régulations et les compromis, pour intégrer le principe des compensations entre les valeurs de nature différente, pour produire des compromis ou des solutions temporaires dans les situations conflictuelles. C'est le cas entre ceux qui défendent, dans les quartiers historiques, un patrimoine matériel bati et ceux qui défendent un patrimoine immatériel dans les pratiques sociales d'usage dans le quartier. Si les transactions sociales aboutissent, celles-ci sont capables d'élargir les capacités de manœuvre des acteurs sociaux pour augmenter les capacités d'interaction et de négociation. Dans ce sens, la transaction sociale représente une méthodologie pour mettre en lumière leurs différences, leurs points communs et leurs possibles arrangements comme une solution re-négociable dans une réalité conflictuelle, en faisant cohabiter, par exemple, une pluralité d'usages dans la réhabilitation d'un quartier.

Les quartiers anciens sont des espaces conflictuels où l'on peut identifier des coopérations entre partenaires, mais aussi entre adversaires, pour réguler leurs conflits sur l'appropriation, l'usage et la mise en valeur de l'habitat dévalorisé. Dans cette logique, la transaction sociale se distingue de la transaction juridique par rapport à la solution, 
car « le contrat social n’est jamais définitivement acquis, il est négociable en permanence, même s'il oppose une grande inertie et évolue lentement» (Blanc, 2009: 27). Dans la transaction sociale, l'accord peut rester informel ; dans certains contextes, la parole peut produire des engagements.

À Strasbourg, par exemple, les actions menées par le Comité d'action pour la réhabilitation sociale de la Krutenau (CARDEK) contre la démolition des ilots complets à la Krutenau, au milieu des années 1970, ont été qualifiées « d'intellectualisme », parce que la discussion contre la rénovation/démolition a été orchestrée par de jeunes étudiants militants de sociologie et d'architecture, dont la politisation a contribué à mettre à l'écart la participation de certains locataires ouvriers. Aussi le CARDEK a mis en place un dispositif de communication, le journal mensuel les «Premières Nouvelles de la Krutenau », où le concept de « réhabilitation surveillée du quartier » essaye de dépasser les autres concepts plus vagues de restauration et de rénovation, en permettant d'établir des alliances et de coopérations entre les anciens locataires et ces jeunes étudiants engagés.

La mobilisation des années 1970 à la Krutenau ne s'explique donc pas suffisamment par une confrontation frontale contre les appareils idéologiques de l’État à la manière des analyses de luttes des classes urbaines de Manuel Castells (1972 ; 1973). Il s'agit plutôt d'une interaction multipolaire entre différents acteurs : militants de gauche, élus, techniciens, locataires, propriétaires. Il existe, en outre, d'autres poles à l'intérieur de chaque groupe. La participation des commerçants dans l'opération est restée très faible, à la différence de l'OPAH qui s'est développée à la Krutenau et dont le role des habitants a permis de mesurer ses effets avec les élus. En fait, les mobilisations des acteurs sont variables et se déroulent à rythmes différents par manque d'instances associatives mobilisatrices des intérets et des valeurs des habitants. Pour les habitants, la qualité de vie n'est pas associée aux espaces verts. Pour la plupart d'entre-eux, les éléments plus attirants sont le bati réhabilité, la proximité du centre-ville, qui n'est pas forcément associée à une proximité du lieu de travail, et la présence d'activités commerciales et culturelles.

De son côté, à Valparaiso, nous avons constaté un rapport de force visible entre formes patrimoniales opposées dans le discours des acteurs nationaux (méso) et locaux (micro) à l'échelle de la Ville-Port. La justification de la revitalisation de la ville a produit une opposition entre la dynamisation et la conservation de biens patrimoniaux, en déclenchant des conflits sur la réappropriation des immeubles de valeur historique. Le système de zones, qui distingue les monuments des immeubles historiques, a produit des tensions dans l'homogénéisation du bâti ancien, mais il a été également un déclencheur des mobilisations de résidents pour l'appropriation et la défense de leur patrimoine bati et des usages de quartier résidentiel. La modification du Plan Régulateur communal a permis la régulation de la « cinquième façade », en régulant le «droit à vue » ou à la « co-propriété de l'œil » selon le MINVU. 
Le débat culturel se concentre aujourd'hui sur la protection, la gestion et la défense du patrimoine, tantốt comme un bien à consommer, alimentant la nostalgie du passé, tantot comme une revendication identitaire ou une prise de conscience de la diversité culturelle (Choay, 1992). La mise en valeur du patrimoine devient ainsi paradoxale : si les enjeux se concentrent seulement sur le báti, muséifiant des quartiers à des fins économiques, elle se fait aux dépens de l'expertise du quotidien fondée sur un savoir-faire lié à la pratique des habitants dans leur territoire, tandis qu'elle pourrait faire partie d'une patrimonialisation populaire basée sur l'expertise profane.

\subsection{Les enjeux de reconnaissance et méconnaissance du patrimoine bâti}

La transaction constitue un type de rapport social qui aboutit à certains accord ou plutôt compromis entre les positions inégales des acteurs. Les transactions sociales permettent de se situer à trois niveaux sociologiques : micro, méso et macro. Elles portent sur l'analyse des couples de tensions, comme éléments structurants de la ville et des quartiers : l'identité et l'altérité, l'ancien et le moderne, le patrimoine matériel et le patrimoine immatériel. Les transactions sociales permettent de prendre en considération les dynamiques socio-spatiales de reconnaissance des habitants et de leur territoire, en intégrant d'autres acteurs et leurs positions, notamment les élus, les promoteurs immobiliers et/ou les experts/techniciens.

Une nouvelle configuration des processus transactionnels se produit dans les processus de valorisation et de dé-valorisation patrimoniale, dont les produits transactionnels portent sur la reconnaissance des habitants et de leur espace. Les processus de patrimonialisation déclenchent la mobilisation des acteurs, mais celle-ci peut produire différentes figures d'acteurs, en fonction de leur degré de conscience dans la connaissance du patrimoine. En fait, l'acteur social se définit par une tension entre l'individuel et le collectif, en suivant deux axes opposés : subjectivité/intersubjectivité et reconnaissance/méconnaissance, ou connaissance déformée. Le schéma 1 montre ces deux positions : d'un côté, la conscience subjectivée et intersubjective, qui s'explique par une tension entre identité et altérité; de l'autre, la reconnaissance et méconnaissance du patrimoine bâti, qui s'explique par une tension entre l'inconnu individuel et le connu socialement travaillé. 


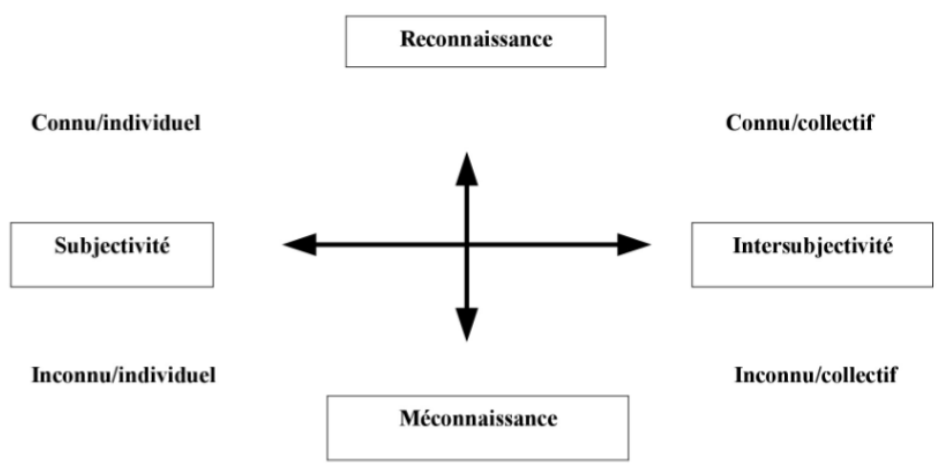

Schéma 1 : les degrés de conscience dans la connaissance du patrimoine

Les degrés de conscience dans la connaissance du patrimoine produisent une tension ( $c f$. schéma 1) entre la conscience subjective et individuelle d'une part, et la conscience intersubjective et collective de l'autre. La reconnaissance est définie comme l'élargissement de la conscience individuelle. Ce schéma s'inspire des travaux d'Axel Honneth: les individus méprisés sont obligés de «lutter pour la reconnaissance » (Honneth, 2002). Il existe des décalages entre la reconnaissance d'un bâti ancien par un expert qui étudie l'immeuble pour le classer et la reconnaissance du bâti par celui qui l'habite. On peut identifier deux types de reconnaissance, l'expertise savante et l'expertise profane. Les éléments que l'architecte reconnaît et valorise ne sont pas les mêmes que ceux que le résident reconnaît et valorise (ce passage où élargissement permet la construction de l'individu comme acteur, membre d'une «Communauté patrimoniale »). Cette communauté regroupe ceux qui sont d'accord sur la défense d'un bien patrimonial, même s'ils le font pour des raisons opposées, en constituant un ensemble de «régulations conjointes ponctuelles» (Reynaud, 1979). Par exemple, les funiculaires à Valparaiso sont des monuments historiques, mais aussi un moyen de transport nécessaire pour les habitants des collines.

On peut aborder la patrimonialisation et la réhabilitation des quartiers anciens à partir les représentations et les discours des acteurs sociaux, par exemple : l'agent de tourisme, l'aménageur, le politicien, le conservateur de musée, le promoteur immobilier, etc. Elle requiert une régulation conjointe pour aboutir à un compromis qui est le résultat des règles acceptées par la multiplicité des acteurs, en constituant «un ensemble raisonnablement cohérent» (Reynaud, 1979). La transaction sociale se situe entre la décision individuelle et la décision collective, lorsque les oppositions des acteurs sociaux structurent des couples de tension. Par exemple, lors d'une opération de l'amélioration d'une façade, on peut identifier des couples de tension s'opposant entre 
la dimension matérielle d'un immeuble et l'immatérialité qui reste dans les représentations sociales de cet immeuble pour les résidents et voisins du quartier (cf. schéma 2 ci-dessous)

Schéma 2 : Vision partielle de l'origine des transactions dans la régulation d'un conflit patrimonial

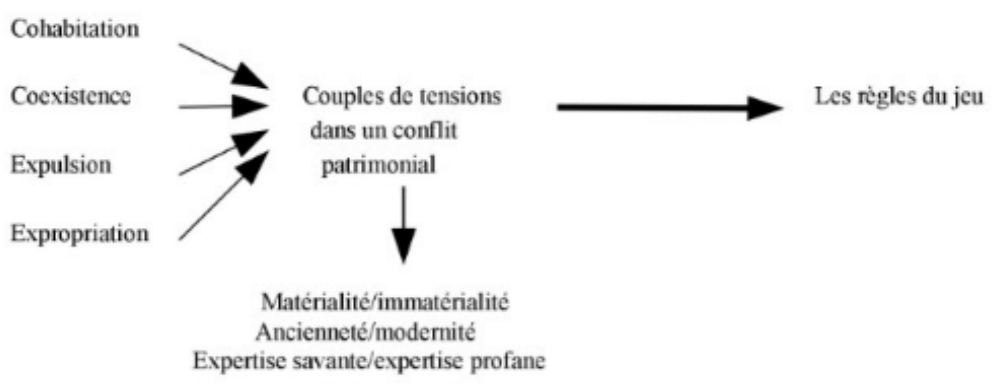

Dans le cas de Valparaiso, le conflit patrimonial a deux volets. D'abord, le système économique néolibéral au Chili permet à l'économie du patrimoine d'occuper une place plus importante que sa conservation. Les monuments et d'autres biens sont renommés comme «biens culturels» auxquels on rajoute une valeur patrimoniale «marchande» à rentabiliser. C'est le cas des funiculaires, des musées et des immeubles (Soto, 2014). Si la rentabilité du bien culturel ne produit pas les résultats attendus, son usage pourra être changé au profit d'un autre. Ensuite, l'image de ville patrimoniale a produit une surexploitation du concept de patrimoine. Aujourd'hui, tout est patrimonial à Valparaiso, même les chiens de la rue, les arbres ou les escaliers ce qui produit des conflits d'usage et des tensions entre deux modèles: celui d'une ville portuaire et celui d'une ville touristique.

Le paradoxe de la condition patrimoniale de Valparaiso réside dans le fait d'articuler la dynamisation économique sur une survalorisation des «biens culturels» dans leur matérialité réhabilitée en fonction d'un marché qui veut réinventer le patrimoine dans les immeubles anciens situés surtout au Cerro Alegre et Cerro Concepción. Dans cette approche, la revalorisation de biens culturels n'assure pas de consensus entre l'objet et la symbolique du patrimoine, comme le soulignent certains experts locaux : «Les biens patrimoniaux ne doivent pas être considérés seulement comme des objets matériels, sinon comme des ressources identitaires et symboliques de la ville pour favoriser les interactions entre les acteurs et la revitalisation du tissu social » (Andueza, 2010). Dans ce contexte, les formes matérielles deviennent des « objets patrimoniaux » à réhabiliter et à restaurer en revalorisant le patrimoine bâti d'un quartier ancien. Même si elles n'ont pas répondu aux attentes, les subventions de réhabilitation ont produit des effets sur les morphologies physique et sociale du Cerro Alegre et du Cerro Cordillera.

Dans le cas de Strasbourg, le centre-ville représente une image historique plus reconnue dans les représentations du patrimoine. En fait, l'image de «ville médiévale» qui 
s'impose dans la Grande Île de Strasbourg est celle d'un patrimoine bati valorisé qui met à l'écart les traces d'une période industrielle antérieure. C'est l'exemple des quartiers péricentraux de la Krutenau et de la Gare. Ces deux quartiers se caractérisent par une cohabitation des identités, entre quartier d'étudiants et quartier de petits-bourgeois, où les traces des quartiers ouvriers sont encore visibles, en attendant de nouveaux usages. Ainsi, La Krutenau et la Gare, ou plus précisément le sous-quartier de l'Académie et le Faubourg de Saverne ont connu des processus de mutations et de passages entre la prolétarisation et l'embourgeoisement. Dans ces deux sous-quartiers, l'identité est le produit d'un rapport de forces entre l'identité collective positive de quartier ouvrier et d'une identité collective négative lorsque le quartier commence à être démoli.

\section{Conclusion}

L'analyse sociologique portant sur les villes patrimonialisées contribue au regard critique sur la politique patrimoniale et les dispositifs d'amélioration de l'habitat ancien. Les concepts de conflit et de forme chez Simmel constituent une approche intéressante à poursuivre afin de comprendre le rapport entre les structures fonctionnelles et les espaces socio-affectifs dans les villes patrimonialisées. À l'échelle locale, le patrimoine présente un intérêt économique à long terme. Si la marchandisation du patrimoine bâti recherche un bien offrant une rentabilité immédiate, la patrimonialisation préfère combiner rentabilité économique à long terme et valeur culturelle. La marque historique, qui est instituée par l'expertise savante du patrimoine dans l'exemple du label de Patrimoine Mondial de l'Humanité, veut "préserver » les intérêts économiques du bien dans une optique de longue durée. Il s'agit d'une dynamique sociale qui fait émerger le conflit comme producteur de lien social.

En effet, l'inclusion des concepts de conflit et de la forme simmelienne dans l'analyse de la sociologie urbaine contribue à une discussion autour des espaces contemporains de la vie collective et de la démocratie participative dans les villes patrimonialisées. La patrimonialisation locale est un défi pour la politique municipale qui débouche sur la production identitaire d'« authenticité » de la ville entière, ou seulement d'une partie. En même temps, la politique patrimoniale locale est le résultat de l'homogénéisation internationale de la monumentalité et de la conservation patrimoniale élaborée par l'expertise savante de l'UNESCO. Ainsi, tant Strasbourg comme Valparaiso ont été classées grâce à leurs valeurs « universelles » d'authenticité répondant à la normalisation internationale de la conservation patrimoniale. Ces valeurs mondialisées représentent des enjeux pour les acteurs locaux qui souhaitent conserver et revaloriser leurs « singularités authentiques » ou plutôt, pour reprendre l'expression de Raymond Ledrut (1973), la personnalité culturelle du lieu. 
Particulièrement, la notion du conflit chez Simmel marque le point de départ de la transaction sociale comme paradigme méthodologique pour comprendre la complexité dans les jeux d'acteurs sociaux et le rôle du conflit dans la production des compromis pratiques comme arrangements provisoires entre des acteurs sociaux. Les acteurs concernés par la revalorisation des quartiers anciens péricentraux sont nombreux : propriétaires et locataires, gouvernements locaux et nationaux, acteurs économiques (promoteurs immobiliers, entreprises du batiment, industrie touristique, artisans et commerçants du quartier, etc.), experts locaux et internationaux ainsi que de nombreux intermédiaires (architectes, associations de défense), etc. La mobilisation de ces acteurs est le fait saillant mis en lumière par l'analyse des processus de patrimonialisation. Ils participent à des jeux multipolaires au sein de ce que nous appelons la " communauté patrimoniale», traversée et structurée par des tensions permanentes et des conflits. Entre les acteurs, il y a à la fois des conflits d'intérets et des conflits de valeurs. Il peut y avoir des alliances, mais elles sont susceptibles d'etre remises en cause. La tension entre les formes matérielles et immatérielles du patrimoine est irréductible ; elle ne peut donner lieu qu'à des compromis instables et provisoires, à renégocier en permanence (Blanc, 1992). Ceci apparaît aussi bien à Valparaiso qu'à Strasbourg : le patrimoine batti est l'objet de conflits, de négociations et d'échanges dans les quartiers étudiés. Les conflits analysés montrent que la transaction sociale est un paradigme heuristique fécond et complémentaire pour analyser l'ordre social et comparer les processus de patrimonialisation dans des contextes différents, où le conflit irréductible est au cœur de la construction d'un projet collectif.

Par exemple, à Strasbourg, la cohabitation de «ville médiévale» et de «ville moderne » trouve un point d'intérêt dans la politique patrimoniale de la Ville qui est en train de mettre en valeur le quartier allemand, Neustadt, dans son immatérialité comme quartier « symbole » de la tolérance strasbourgeoise. Une valeur immatérielle du patrimoine, dans la stratégie patrimoniale de la Ville, qui porte des intérêts marchands sur la valeur matérielle du patrimoine dans les quartiers péricentraux, que l'on peut analyser en se basant sur les notions simmeliennes.

Chez Simmel, l'articulation entre la distance et la proximité, entre le centre-ville et les quartiers péricentraux devient véritablement une stratégie de valorisation et de dévalorisation, de distance sociale et de proximité spatiale au sein des quartiers. La valorisation patrimoniale du centre-ville signifie la reconnaissance d'une forme patrimoniale homogène qui exclut les autres formes coexistantes - reflets d'autres époques dans les quartiers péricentraux. À Strasbourg et à Valparaiso, une certaine vie sociale patrimoniale reste dans l'ombre de la non-reconnaissance, mais pour qui ?

\section{Bibliographie}

Adeus (2002a), Propositions d'orientations et d'actions. Programme local de l'Habitat de la communauté urbaine de Strasbourg, Strasbourg. 
Adeus (2002b), "Ségrégation spatiale, ségrégation sociale », Dimension Villes, n³5, Strasbourg.

ANDUEZA P. (2010), « La política de activación patrimonial en Valparaíso: critica y propuesta », documento presentado al primer encuentro de la Sociedad de Políticas Públicas, Universidad Adolfo Ibáñez, Santiago enero 2010.

Authier J-Y., Bidou C. (2008), « La gentrification urbaine », Espaces et Sociétés, nº 132-133, p.13-21.

Benavides J., Pizzi M., Valenzuela M. P. (1994), Ciudades y Arquitectura Portuaria, Valparaíso, Editorial Universitaria.

Bidou-Zachariasen C. (dir.), (2003, Retours en ville, Paris, Descartes \& Cie.

Blanc M. (2009), La transaction sociale: genèse et fécondité heuristique», Pensée Plurielle, $\mathrm{n}^{\circ}$ 20, p. 25-36.

BLANC M. (2006), « Conflits et transactions sociales : la démocratie participative n’est pas un long fleuve tranquille », Sciences de la société, $\mathrm{n}^{\circ} 69$, p. 25-37.

Blanc M. (dir.) (1992), Pour une sociologie de la transaction sociale, Paris, L'Harmattan.

BouRdin A. (1984), Le patrimoine réinventé, Paris, Presses universitaires de France.

BuRgUeSS E. (1967[1925]), "The Growth of the City. An Introduction to a Research Project» in R. Park, E. Burguess, R. McKensie, The City, Chicago, University of Chicago Press, p. 47-62.

CASTELls M. (1973), Luttes urbaines et pouvoir politique, Paris, Maspero.

CASTELLS M. (1972), La question urbaine, Paris, Maspero.

CASTILLO O. (2005), « La ciudad-puerto de Valparaíso como patrimonio arquitectónico e histórico-cultural de la humanidad », Revista archivum año IV, nº 5, p. 27-39.

CHOAY F. (1992), L’Allégorie du patrimoine, Paris, Seuil.

CoING H. (1966), Rénovation urbaine et changement social, Paris, Éditions ouvrières.

De Certeau, M. (2005[1990]), L'invention du quotidien, t. 1 : Arts de faire, Paris, Gallimard,.

Elias N., Scotson J. (2001[1965]), Les logiques de l'exclusion, Paris, Pocket.

Foessel G., Coltat F. (1989), La Krutenau perdue et retrouvée. Strasbourg, Contades.

HonNETH A. (2002[1992]), La lutte pour la reconnaissance, Paris, Cerf.

JEUDY H-P. (1990), « Introduction », in H.-P. JEUdy (dir.), Patrimoine en folie, Paris, Maisons des sciences de l'homme, p.1-10.

LEDRUT R. (1973), Les images de la ville, Paris, Anthropos. 
LE GofF J. (1988), Histoire et mémoire, Paris, Gallimard.

LEVY J-P. (1990), « Quartiers anciens ou centres-villes, quatre scénarios pour l'avenir », in J.-P. LEVY (dir.), La réhabilitation des quartiers anciens et de l'habitat existant : Acteurs, procédures, effets et conséquences sociales, Toulouse, Presses universitaires du Mirail, p.161-173.

LINOSSIER R., RUSSEIL S., VERHAGE R., ZEPF M. (2004), « Effacer, conserver, transformer, valoriser. Le renouvellement urbain face à la patrimonialisation », Les Annales de la Recherche Urbaine, $\mathrm{n}^{\circ}$ 97, p. 23-26.

MAURICE M. (1989), "Méthode comparative et analyse sociétale. Les implications théoriques des comparaisons internationales », Sociologie du travail, vol. $31, \mathrm{n}^{\circ} 2$, p. 175-191.

REMY J. (dir.). (1995), Georg Simmel : Ville et modernité, Paris, L’Harmattan, p. 149-176.

REMY J. (1998), «La transaction sociale, formes de sociabilité et postures méthodologiques » in M.-F. Freynat, M. BLANC, G. PinEAU (dir.), Les transactions aux frontières du social, Lyon, Chronique sociale, p. 20-42.

REMY J. (1999), "La ville: architecture spatiale et univers d'intercompréhension », Recherches sociologiques, vol. $30, \mathrm{n}^{\circ} 1, \mathrm{p} .177-183$.

REynaud J-D. (1979), «Conflit et régulation sociale. Esquisse d'une théorie de la régulation conjointe », Revue française de sociologie, vol. 20, p. 367-376.

SAYEZ L. (2001), Valparaíso. Lugares, nombres y personajes, siglos XVI-XXI, Valparaíso, Puntángeles, Universidades de Playa Ancha y de Santiago de Chile.

SERFATY-GARZONP. (1992), "Sens du présent et visibilité du passé à propos des transformations de la signification de la sauvegarde du patrimoine bâti », SocioEnvironmental Metamorphoses: Builtscape Landscape Ethnoscape Euroscape,Marmaras, Chalkidiki, Greeces 1992, p. 372-380.

Simmel G. (2003[1908]), Le Conflit, Paris, Circé/Poche.

Simmel G. (2006[1909]), « Esthétique et sociologie » in G. SimmEL, La Tragédie de la culture et autres essais, Paris, Rivages poche, p. 129-138.

Simmel G. (2009[1903]), « Métropoles et mentalité » in Y. GrafMEYeR, I. JoSEPH L'École de Chicago. Naissance de l'écologie urbaine, Paris, Champs/essais, p. 61-77.

Soто M. (2014), « Les paradoxes de la mise en valeur des funiculaires de Valparaiso : le conflit entre patrimonialisation matérielle et immatérielle ", L’Homme et la Société, $\mathrm{n}^{\circ}$ 191, p. 139-156.

Soto M. (2011), «Reconnaissance et transaction sociale dans l'espace urbain patrimonial: processus de valorisation et dévalorisation, Strasbourg et 
Valparaiso », in P. HAMman, J.-Y. CAUSER (dir.), Ville, environnement et transactions démocratiques, Bruxelles, PIE-Peter Lang, p. 111-131.

TREPOS J-Y. (1996), « Prudences expertes et aménagement urbain », Espaces et Sociétés, $\mathrm{n}^{\circ}$ 84-85, p. 203-226.

Trivelli P., Nishimmura Y. (2010), The sustentability of urban heritage preservation: interventions to support economic and residential investments in urban heritage areas of Latin America and the Caribbean (RG-T1620): case study Valparaíso, Santiago, Pablo Trivelli y Cia Ltda.

VERMEERSCH L. (2003), «La centralité urbaine comme patrimoine ou les nouvelles formes de la reconquête des centre-villes aux États-Unis, l'exemple de Providence (Rhode Island) », in M. GRAVARI BARBAS, S. GUICHARD-ANGUIS (dir.), Regards croisés sur le patrimoine dans le monde à l'aube du XXIe siècle, Paris, Presses de l'Université de Paris-Sorbonne, p. 609-621.

WAISBERGM. (1992), La arquitectura religiosa de Valparaíso, siglo XVI-siglo XIX, Valparaíso, Editorial Universidad de Valparaíso. 